\section{Adrenaline in anaphylaxis: overtreatment in theory, undertreatment in reality}

\author{
Peter Storey, Penny Fitzharris
}

'Anaphylaxis is a clinical emergency, and all healthcare professionals should be familiar with its management'. ${ }^{1}$ Few health professionals would disagree with this opening sentence of new guidelines from the European Academy of Allergy and Clinical Immunology (EAACI), which aim to provide evidence-based recommendations for recognition, risk assessment, and management of patients who have experienced, are experiencing or are at risk of experiencing anaphylaxis. This care requires correct recognition of anaphylaxis, appropriate acute management and optimal long-term care: all important but distinct skills.

Guidelines clarify what treatment should be given to whom and when. It would be hoped that, over time with codification of best practice, doctors' abilities to accurately recognise and appropriately treat anaphylaxis would improve. However, Plumb and colleagues found, using brief written case scenarios, that junior doctors today seem to be no better at correctly identifying the clinical need for, and correct dose and route for administration of, adrenaline (epinephrine) than their predecessors a decade earlier. ${ }^{2}$

All doctors in this recent study recognised adrenaline as the appropriate treatment for a case scenario that clearly described anaphylaxis. However, many (21-82\%) also selected adrenaline as treatment for scenarios describing situations in which adrenaline would not be appropriate-for example, inhalation of peanut or acute urticaria with no other system involvement. Little seems to have changed over time. A decade earlier, all junior doctors at the same hospital tested using the same scenarios selected adrenaline as treatment of choice for anaphylaxis, but some (10-56\%) also indicated that they would use adrenaline for inappropriate scenarios.

Similar results have been found elsewhere in the world. In our recent

Immunology Department, Auckland City Hospital, Auckland, New Zealand

Correspondence to Dr Penny Fitzharris, Immunology Department, Auckland City Hospital, Park Road, Auckland 1111, New Zealand; pennyf@adhb.govt.nz unpublished study from Auckland, all 22 postgraduate year 1 doctors tested using similar scenarios selected adrenaline to treat anaphylaxis, but 27-91\% would also have used adrenaline inappropriately.

A questionnaire study of doctors and nurses in a large Singapore hospital indicated good recognition of anaphylaxis but also a tendency to overdiagnosis. ${ }^{3}$ Anaphylaxis is typically rapid in onset, with involvement of more than one system: airway, breathing or circulatory and usually, but not always, skin or mucosal changes. In the Singapore study, ${ }^{3} 89 \%$ of emergency department doctors, the majority of whom had previously seen at least one case of anaphylaxis, chose adrenaline correctly for written scenarios describing symptoms that involved two systems. However, as in the UK study, there was a tendency to recommend adrenaline in patients with single organ (skin) involvement.

It is crucial that the correct dose of adrenaline is delivered by the correct route. Incorrect use of intravenous adrenaline may endanger patients' lives. While there was an improvement in both correct choice of dose and route of administration in the decade between the two UK studies, a significant number of doctors in all three studies selected the wrong dose and the wrong route. Given the evident lack of knowledge regarding the correct dose and route, easy access to a simple anaphylaxis algorithm, appropriate-strength (1:1000) adrenaline and the necessary syringes and needles should be provided on all resuscitation trolleys. Such an 'anaphylaxis box' was recently introduced in Auckland City Hospital and has been well received and considered very useful by resuscitation teams (boxes 1 and 2). A similar suggestion to place advance life support (ALS) anaphylaxis guidelines on resuscitation trolleys was made after another study of first- and second-year UK doctors in 2008 identified that even junior doctors who had completed ALS training had poor knowledge of adrenaline use and dose. ${ }^{4}$

It seems that, when tested in this way, doctors' ability to discriminate between clinical descriptions of any complexity is imperfect and theoretically, at least, indicates intended overuse of adrenaline.
Box 1 Recognise, treat, reassess

- Recognition: if a patient has acute onset airway and/or breathing problems, or hypotension especially if the skin is affected (eg, urticaria), strongly consider treating as anaphylaxis

- Treatment: adrenaline $0.5 \mathrm{mg}$ intramuscularly lateral mid thigh (adult) - repeat after $5 \mathrm{~min}$ if no response.

- Reassess and monitor closely. Use oxygen, intravenous crystalloid, $\beta$-agonist according to clinical need. Patient may be semi-recumbent if wheezing or vomiting, otherwise lie flat. Get help. Don't panic.

However, this does not reflect what happens in practice. Delayed administration of adrenaline in patients with anaphylaxis has been shown to be a risk factor for poor outcome, ${ }^{5} 6$ and retrospective audits show underuse of adrenaline to be common. ${ }^{7}$ Data from the UK register of fatal anaphylactic reactions shows non-administration of adrenaline happens in 39\% of anaphylactic fatalities regardless of the precipitant. Although overdiagnosis of anaphylaxis has been found to contribute to harm to patients, this is rarer than omission of adrenaline. ${ }^{8}$

It is not clear why adrenaline is withheld in the face of anaphylaxis. Perhaps there is an element of panic, both in patients experiencing escalating life-threatening symptoms and in their attendant health professionals who do not often deal with such urgent 'high-stakes' clinical problems. Doctors may be unfamiliar with intramuscular administration of adrenaline. Possibly, there are some concerns about the potential

\section{Box 2 Recommendations}

- Have a clearly labelled anaphylaxis kit, with adrenaline, needles, syringes and a simple treatment algorithm available as a separate item in your resuscitation equipment.

- Teach all clinical staff, including nurses, anaphylaxis recognition and treatment as part of advance life support training.

- Consider use of simulation/on-site anaphylaxis scenario practice in high-risk areas.

- Consider use of online training. 
risks of adrenaline, although all guidelines indicate that risks of giving adrenaline in the correct dose by the correct route are very modest.

Recognition of the underlying clinical condition is clearly an essential first step for appropriate treatment (see box 1). Guidelines are helpful, but may understate subtleties, variability and complexities of clinical presentations, although the UK Resuscitation Council guidelines are commendable for their functional approach. ${ }^{9}$ Surprisingly, there is no universally accepted definition of anaphylaxis. Definitions used in the three major anaphylaxis guidelines, from AAAAI/ACAAI, ${ }^{5}$ World Allergy Organisation $^{6}$ and EAACI, ${ }^{1}$ are noted in a recent 'international consensus' by Simons and colleagues ${ }^{10}$ to 'all include the concepts of a serious, generalised or systemic, allergic or hypersensitivity reaction that can be lifethreatening or fatal'.

Responses to such deficiencies in practice usually include the suggestion of more 'education and training'. There is recognition of the need for improved training in the recent international consensus. ${ }^{10}$ which states 'In all countries, improved training of healthcare professionals to recognise and treat anaphylaxis is needed, and the validated clinical criteria for anaphylaxis diagnosis need to be operationalised in order to optimise their usefulness'. Unfortunately, no practical advice on how best to do this for junior doctors and other healthcare providers was offered.

Thus, while studies suggest that doctors know that adrenaline is required in anaphylaxis, this knowledge is often not translated into practice. It would seem that traditional approaches to education are not enough: half the doctors in the Plum study had completed ALS training. Perhaps it is more pertinent that most had not worked in an emergency department and so are unlikely to have seen a patient with anaphylaxis. We need to rethink how we train doctors and nurses in the care of all aspects of management of this lifethreatening condition. Modern technology and modern educational techniques could hold the key.

Simulation is one possible approach that may provide more effective training in management of anaphylaxis. When junior doctors working in an Australian emergency department were assessed using a simulated child with anaphylaxis, their actions and responses in this simulated environment were much more in keeping with what is known about actual practice than the responses to written scenarios in the other studies. Only 50\% of these doctors administered adrenaline in scenarios that simulated definite anaphylaxis and were more likely to administer adrenaline if they had had previous advanced resuscitation training or had more years of training. While this study did not look at the subsequent impact of the training, all participants believed that the simulated mini-clinical examination (mini-cex) format used in this study had improved their ability to recognise and treat anaphylaxis. ${ }^{11}$

Simulation offers the opportunity to train and assess doctors in a range of presentations of anaphylaxis and so to reveal the complexities not readily understood from guidelines. In the Australian study, junior doctors were presented with two simulated scenarios-one with and one without hypotension. Adrenaline use was more common with the presence of hypotension, which is of some concern as food-induced anaphylaxis typically results in respiratory distress rather than hypotension. It would also be possible to include training on the actual administration of adrenaline (see box 2).

Research on retention of resuscitation skills has shown deterioration in a number of skill areas in the weeks to months after training on a resuscitation programme, accompanied by a decline in clinician confidence. Greater access to and hands-on training opportunities have been shown to aid retention of basic life support skills. ${ }^{12}$ The same is likely to apply to anaphylaxis training, so 'booster' sessions providing practice opportunities would improve recognition and treatment of anaphylaxis, preventing deterioration of these important skills.

Anaphylaxis is a relatively infrequent (approximately 1 in 3500 UK individuals per annum) life-threatening, but usually treatable, condition. ${ }^{13}$ We know that some patients die because they are not given adrenaline soon enough or at all or are given it by the wrong route. This is a long-standing and international problem. In our view, more effective training is the key to better outcomes. Doctors, especially those in emergency departments, need to be skilled and confident in the care of these patients. We suggest the development and use of simulated training programmes to provide a practical but safe approach to both training and assessment. Guidelines are important but of limited usefulness when there is a gap between knowledge and practical application. Closing this gap should lead to better patient outcomes.

Competing interests None.
Provenance and peer review Commissioned; internally peer reviewed.
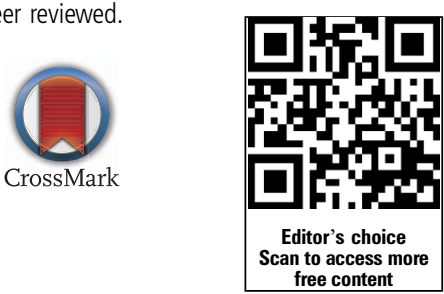

To cite Storey P, Fitzharris P. Postgrad Med J 2015;91:1-2.

Accepted 8 December 2014

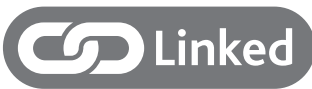

http://dx.doi.org/10.1136/postgradmedj-2013132181

Postgrad Med J 2015;91:1-2.

doi:10.1136/postgradmedj-2014-133185

\section{REFERENCES}

1 Muraro A, Roberts G, Worm M, et al. Anaphylaxis: guidelines from the European Academy of Allergy and Clinical Immunology. Allergy 2014;69:1026-45.

2 Plumb B, Bright P, Gompels M, et al. Correct recognition and management of anaphylaxis: not much change over a decade. Postgrad Med J 2015;91:3-7.

3 Ibrahim I, Chew BL, Zaw WW, et al. Knowledge of anaphylaxis among Emergency Department staff. Asia Pac Allergy 2014;4:164-71.

4 Gopalikrishnan S, Alexander R. Anaphylaxis: junior doctors' knowledge of guidelines. Resuscitation 2009;80:383-4.

5 Lieberman P, Nicklas RA, Oppenheimer J, et al. The diagnosis and management of anaphylaxis practice parameter: 2010 update. J Allergy Clin Immunol 2010;126:477-80

6 Simons FER, Ardusso LRF, Bilo MB, et al. for the World Allergy Organisation. World Allergy Organisation guidelines for the assessment and management of anaphylaxis. J Allergy Clin Immunol 2011;127:587-93, e1-e22.

7 Russell S, Monroe K, Losek JD. Anaphylaxis management in the pediatric emergency department: Opportunities for improvement. Pediatr Emerg Care 2010;26:71-6.

8 Pumphrey R. Lessons for management of anaphylaxis from a study of fatal reactions. Clin Exp Allergy 2000;30:1144-50.

9 UK Resuscitation Council. https://www.resus.org.uk/ pages/reaction.pdf (accessed 7 Dec 2014).

10 Simons FER, Ardusso LRF, Bilo MB, et al. International consensus on (ICON) anaphylaxis. World Allergy Organ J 2014;7:9.

11 O'Leary FM, Hokin B, Enright K, et al. Treatment of a simulated child with anaphylaxis: an in situ two-arm study. J Paediatr Child Health 2013:49:541-7.

12 Curran V, Fleet L, Greene M. An exploratory study of factors influencing resuscitation skills retention and performance among health providers. J Contin Educ Health Prof 2012;32:126-33.

13 Stewart AG, Ewan PW. The incidence, aetiology and management of anaphylaxis presenting to an accident and emergency department. QJM 1996:89:859-64. 